\title{
Analysis of Therapy Transcripts using Natural Language Processing
}

\author{
Sarah Hawa, Shriya Akella, Shrishti Kaushik, Vrushali Joshi, Dhananjay Kalbande
}

\begin{abstract}
Mental health plays an integral part in leading a healthy life and having a positive outlook. This impacts our behavior, thought process, and actions and therefore it's important to identify and detect mental disorders in an early stage as it's effects can have a lasting influence on one's life. According to WHO, one in four people get affected by mental health disorders and currently 450 million people suffer from such conditions. Natural Language Processing can be a useful tool to analyze the trends in therapy transcripts. They can be further trained and optimized to derive useful insights and predict plausible future trends. Our proposed system analyses therapy transcripts and classifies it as 'Early signs of depression' and 'Serious after-effects of prolonged depression' based on the nature of the responses. Our system uses three different classifiers- Naïve Bayes, Support Vector Machine, and Logistic regression as well as two different victories- TF-IDF and Count, to classify the text into these categories. This proposed system will not only help patients in identifying their symptoms but will also help therapists and researchers in gathering a large amount of data which could be used in predictive analysis, diagnosis and understanding the patient. Such research will pave the way for improving counselling and therapy sessions and be a very essential analysis tool for therapists.
\end{abstract}

Keywords: Naïve Bayes, TF-IDF, Vector Machine.

\section{INTRODUCTION}

Mental health denotes a person's state of psychological well being. A mentally healthy person is capable of utilizing their cognitive abilities to perform the usual functions that their body and surroundings demand. Mental illnesses can be treated through counselling, sometimes accompanied with prescribed medicine. According to WHO, one in four people get affected by mental health disorders and currently 450 million people suffer from such conditions [1] . Mental illnesses such as depression and anxiety can have dire consequences as they can lead to suicide, if not addressed and treated in due time. It becomes very crucial to identify such mental illnesses at an early stage. To make the process of identification efficient and quick, NLP based prediction can be utilised. With the help of a thorough analysis of therapy transcripts, early signs of mental illness such as depression, anxiety can be recognised.

Revised Manuscript Received on August 15, 2020.

* Correspondence Author

Sarah Hawa*, Department of Electronics and Telecommunication, Sardar Patel Institute of Technology, Mumbai, India. E-mail: sarah.hawa@spit.ac.in

Shriya Akella, Department of Electronics and Telecommunication, Sardar Patel Institute of Technology, Mumbai, India. E-mail: shriya.akella@spit.ac.in

Shrishti Kaushik, Department of Electronics and Telecommunication, Sardar Patel Institute of Technology, Mumbai, India. E-mail: shrishti.kaushik@spit.ac.in

Vrushali Joshi, Department of Electronics and Telecommunication, Sardar Patel Institute of Technology, Mumbai, India. E-mail: vrushali.joshi@spit.ac.in

Dhananjay Kalbande, Department of Computer Science, Sardar Patel Institute of Technology, Mumbai, India. E-mail: drkalbande@spit.ac.in

(C) The Authors. Published by Blue Eyes Intelligence Engineering and Sciences Publication (BEIESP). This is an open access article under the CC BY-NC-ND license (http://creativecommons.org/licenses/by-nc-nd/4.0/)
This analysis can further be used to obtain useful insights and predict future trends. Our proposed NLP algorithm analyses the transcripts of therapy sessions in order to perform classification based on the nature of responses. The data set consisting of therapy transcripts from counselling sessions is first analyzed to gain insight about the various characteristics of the transcripts and accordingly determine the features and scope of our algorithm. Then, the text is preprocessed in order to make it easy to understand for further processing. This is then followed by vectorization which is performed by calculation of TF IDF (Term frequency-inverse document frequency) and making use of Count vectorizer. This text is then classified into 'Early signs of depressions' and 'Serious after-effects of prolonged depression'.

\section{LITERATURE SURVEY}

Mental health has become exceedingly important to maintain a healthy lifestyle and a positive outlook. The awareness regarding mental health issues has gained prevalence in recent times. The system described in this paper talks about the classification performance of various classifiers such as Random Forests (RF), Support Vector Machines (SVM), Linear Discriminant Analysis (LDA), and k-Nearest Neighbor. LDA was considered the most efficient in terms of average generalization errors. One major drawback of LDA is that it only performs well on a smaller number of correlated features. [2] This paper monitors the Twitter feed of 135 Mechanical Turk (MTurk) participants and was compared with validated reports of suicide risk using a machine learning algorithm. The algorithm was able to identify the suicidal cases with $92 \%$ accuracy. The algorithm falls short in terms of identifying linguistic characteristics which is extremely vital in differentiating between various mental health-related causes. [3] A system that conducts emotion detection on Twitter feeds using natural language processing has been described in this paper. The tweets are classified as negative or positive using classifiers such as Support Vector Machine and Naïve Bayes. Multinomial Naïve Bayes model outperforms the Support Vector Machine model. A drawback of this system is the low accuracy caused by the use of short forms. [4] This paper talks about a system that processes Facebook data based on emotional process, temporal process, linguistic style, and all the aforementioned features together using techniques such as decision tree, k-Nearest Neighbor, Support Vector Machine, and ensemble. The Support Vector Machine model has better performance. However, the decision tree has better results for precision, recall, and f1 score. [5]

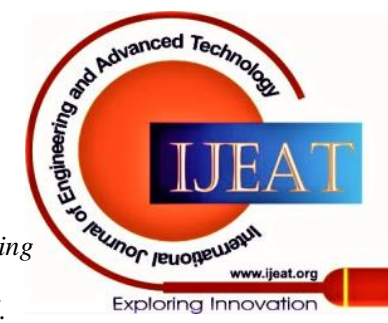




\section{Analysis of Therapy Transcripts usingNatural Language Processing}

The system described in this paper talks about a system to classify 10 subreddit COVID-19-related comments using Natural Language Processing. Linear Discriminant Analysis was used for topic modeling and LSTM was used for sentiment classification of the text. The scope was limited as it was restricted to the English language only. [6] In this paper, a system that makes use of a neural network for online character recognition for recognizing a physician's handwriting. Natural Language Processing is used for formatting and classifying the prescription. This system outperforms an existing solution called MedEx. [7] The " $n$ grams" feature was used in this paper, meaning that predictions were based upon a contiguous sequence of $n$ words that were codified and used as an input to the machine learning algorithm. The system was able to predict the probabilities of suicidal ideation or heightened psychiatric symptoms of the patients. However, while deciding the size of the n-grams, there was a trade-off between higher specificity and lower sensitivity. [8] In this paper, a system that classifies the symptom keyword as 'negative symptoms' or 'catatonic syndrome'. Text Hunter, an information extraction suite, was used for the same. A major drawback of the system was that it was not comprehensive and missed aspects of psychosis presentation. [9] This paper aimed to use natural language processing to perform prediction of psychiatric readmission to promote interventions for this purpose. The natural language processing model used here is a Latent Dirichlet Allocation (LDA) model. This model is used to determine the most relevant and frequent words on specific subjects presented in a text document. The topics considered here for the LDA model were depression, anxiety, trauma, etc. TF IDF (Term frequency-inverse document frequency) was also calculated to recognize the most frequently appearing words and limit their lexicon. SVM was further used for classification and, specificity and sensitivity were used as performance metrics. [10]

\section{METHODOLOGY}

For the implementation of our model, we will be using natural language processing and machine learning classification algorithms to analyse transcripts and predict the nature and seriousness of the patient's mental illness.

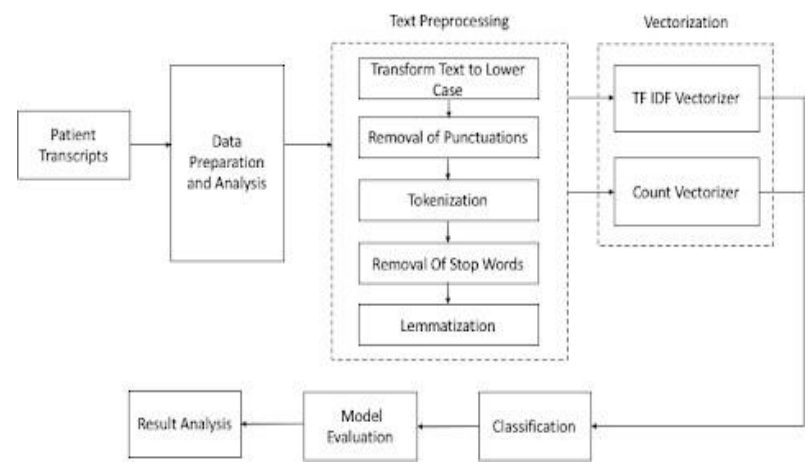

Fig. 1. Block Diagram and System Design

\section{A. Dataset}

The dataset was prepared from transcripts generated from interactions with therapists on an online platform called Counsel Chat. The platform has over 300 plus therapists that provide online assistance and therapy on mental health issues. The dataset consists of 31 mental health related issues that was generated from 317 transcripts. [11] These 31 uniquely identified issues consisted of mental health problems such as depression, anxiety, anger management, substance abuse, domestic violence etc.

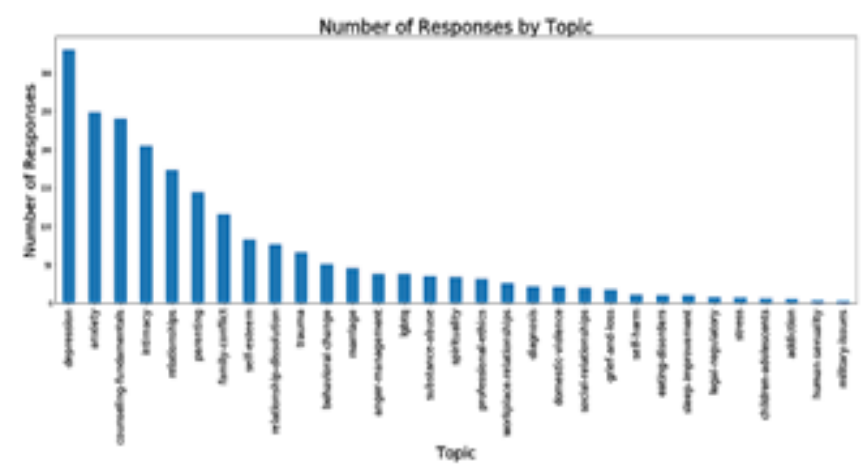

Fig. 2. Topic-wise number of responses

\section{B. Data Preparation and Analysis}

In the above Figure 2 , mental health topics and issues are plotted by the number of responses received from patients. It is observed that most of the mental health related problems and topics revolve around depression, relationship, anxiety, self esteem and family conflicts. On the lower end of the spectrum, topics such as sleep improvement, eating disorders etc have fewer responses and questions.

Another interesting analysis was pertaining to the length of the conversation between therapists and patients. In Figure 3 , it is observed that the average question length was around 54 words whereas the average response length was 170 words long.

Furthermore in Figure 4, when we plot the length of the responses by mental health related topics, we found out that domestic violence, spirituality, workplace relationships, and
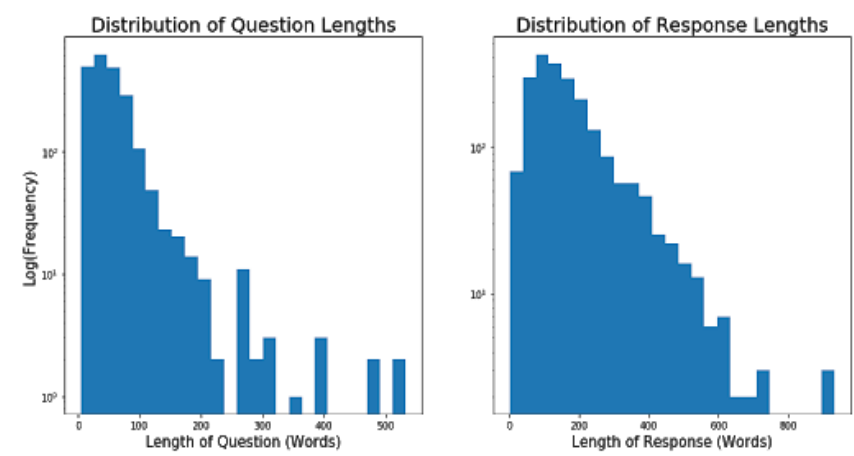

Fig. 3. Distribution of length of questions and responses

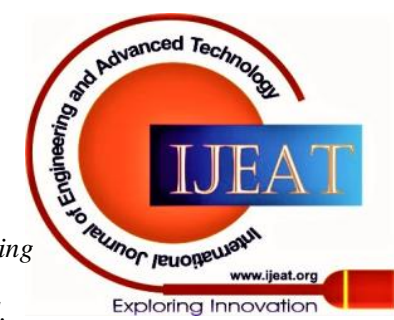




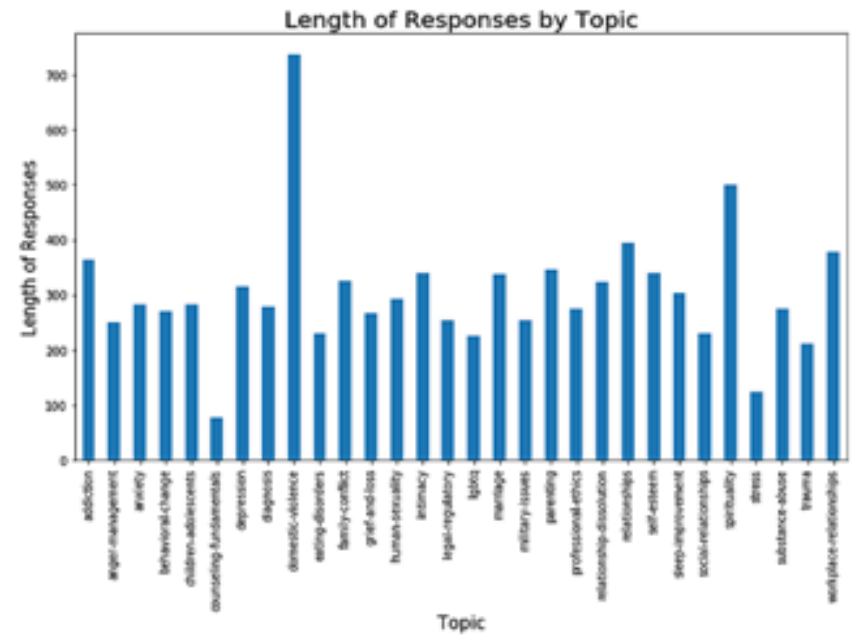

Fig. 4. Length of responses by topic

Addiction record the highest lengths. This denotes that these mental health related concerns are caused by deep-rooted and long term effects of depression and trauma. For data preparation, we classify issues such as depression, anxiety, self esteem, trauma, behavioral change, grief and loss, self harm, stress and anger management under early signs of depression that need immediate intervention as it is easier to tackle mental health issues at an early stage. Issues such as relationship-dissolution, workplace-relationships, domestic-violence, sleep-improvement, substance-abuse, family-conflict, marriage, eating-disorders, relationships, LGBTQ, addiction, legal-regulatory, professional-ethics, military-issues and diagnosis are classified under serious after effects of prolonged depression causing negative impacts on not only the patient but others around them.

\section{Text Preprocessing}

The purpose of text preprocessing is to transform the text in a format that is readable and at the same time understandable by the machine in order to make predictions accurately. It is also essential to select text preprocessing techniques which will work best with the model in order to obtain precise results. For text preprocessing, we will be using the NLTK (Natural Language Toolkit) library.

1) Lower case: The first step is to transform our text into lower case in order to reduce the vocabulary size, list and word count. This is not only necessary due to the case sensitivity of the text but also helps in getting rid of duplicate values of the same word.

2) Removal of punctuation: After converting our text to lowercase, we then proceed to remove punctuation by using string. Punctuation. Removing punctuation aid in normalising the words by avoiding identical versions of the same.

3) Tokenization: After the removal of punctuation, we then transform our text to tokens by using the function word tokenize() from the NLTK library. This function splits the text string into tokens based on the white spaces between words in a sentence. This is an important immediate step before we remove stop words and perform lemmatization and stemming on text.

4) Removal of Stop words: After we tokenize our text, we then remove stop words from our list of tokens. Stop words are nothing but a set of words that occur frequently in text or sentence but their contribution is insignificant for the purpose of text analysis.

5) Lemmatization: After removing stop words, we then perform lemmatization on the tokens. Lemmatization is quite similar to stemming, however it is more intelligent in terms of reducing the word by removing suffixes, prefixes, infixes and circumfixes. After lemmatising we then re-transform the tokens into sentences for the purpose of vectorising by TF- IDF and Count vectoriser.

\section{Vectorization}

In order for the machine to understand and comprehend text, it's extremely important to vectorize the preprocessed text into numbers. This is done by using two methods namely TF IDF and count vectoriser.

1) TF IDF (Term frequency-inverse document frequency): TF IDF weight is an important concept generally used in information recovery and is an important statistical tool to signify, evaluate and rank the importance and relevance of a particular word in a document. This tool comprises two terms: Term frequency (TF) which records the occurrence of a particular word in a document divided by the total word count whereas IDF determines the significance of a word.

2) Count Vectorizer: This vectorizer breaks down a text into a vocabulary of words. It then returns an encoded form of a vector along with the length of the vocabulary and the count of occurrence for each word.

\section{E. Classification}

Once the text has been preprocessed and vectorised, we then train different classifiers to predict the nature and seriousness of the mental health related problem and choose a model that works best for our use case. Thus we will be training our data on the following algorithms:

1) Naïve Bayes: Probabilistic classifiers such as Naïve Bayes which is based on the Bayes' theorem. It's a simple yet extremely efficient and popular method used for natural language processing (NLP) applications.

2) Support-Vector Machines (SVMs): Models based on supervised learning such as support-vector machines (SVMs) which is widely implemented for textual analysis and classification based problems.

3) Logistic Regression: Logistic Regression which is another widely implemented supervised learning algorithm which classifies and makes predictions on probabilities of dependent variables.

\section{F. Model Evaluation}

Once we have preprocessed, vectorised and trained our model with classification algorithms, we test and evaluate the model and its performance in order to see which classification algorithm works best for our problem statement. In order to do this we make use of the following methods such as classification report which summarises the accuracy, precision, recall and F1 score for every algorithm which is calculated using true and false positives, true and false negatives.

Published By: Blue Eyes Intelligence Engineering

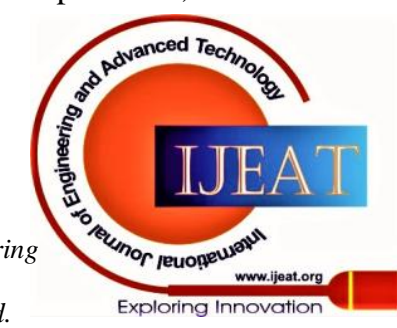




\section{Analysis of Therapy Transcripts usingNatural Language Processing}

The former is then represented pictorially with the help of a confusion matrix. Furthermore the AUC ( area under the curve) is also calculated which is an important metric while dealing with classification problems.
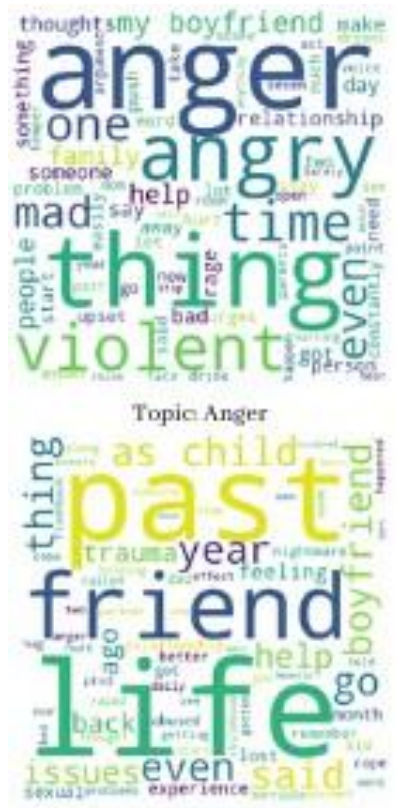

Topie Trauma

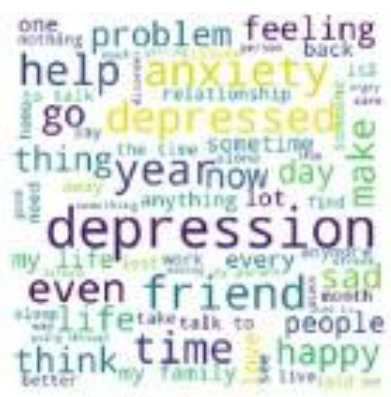

Topic Depression

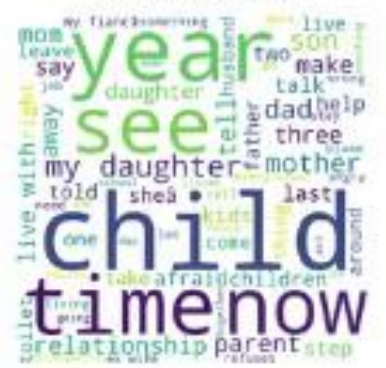

Topic Parenting

Fig. 5. Word cloud visualization for anger, depression, trauma and parenting

topic anger-management.

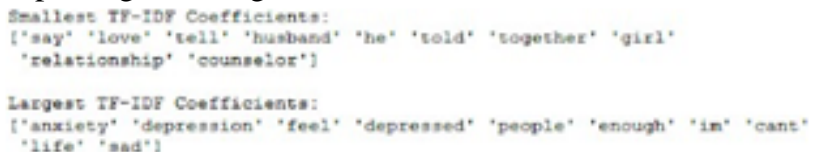

Fig. 6. TF-IDF coefficients

Figure 6 shows ten words with smallest and largest TFIDF coefficients found for the logistic regression classifier. As seen from the figure words like love, relationship, counselor are given small coefficients which seem to belong to the responses labeled as class 0 that is the class considered as the after effects of prolonged depression and words like depression, depressed, anxiety are given large coefficients which seem to belong to the responses labeled as class 1 which is the class considered as the early stage of depression.

\section{G. Model Performance and Analysis}

Table- I: TF-IDF Vectorizer Results

\begin{tabular}{lccclc}
\hline & $\begin{array}{l}\text { Precison Recall f1 } \\
\text { Score }\end{array}$ & $\begin{array}{l}\text { AUC } \\
\text { Score }\end{array}$ & Accuracy \\
\hline $\begin{array}{l}\text { Logistic } \\
\text { Regression } \\
\text { Multinomial }\end{array}$ & 0.80 & 0.79 & 0.79 & 0.79 & 0.82 \\
NB & 0.81 & 0.77 & 0.78 & 0.77 & 0.82 \\
SVC & 0.86 & 0.85 & 0.85 & 0.85 & 0.87 \\
\hline
\end{tabular}

\section{RESULTS}

\section{A. Textual Analysis}

We used word clouds to visualize the transcripts data and see which words are significantly used in topics like depression, trauma, anxiety etc. As seen from Figure 5 , bolder and bigger words that appear in the word cloud are

more significant to that topic. Thus words like depressed, anxiety, sad are used more often in the responses for the topic Precison Recall f1 AUC Accuracy Score Score depression. Similarly, words like anger, angry, violent have been used significantly in the responses classified under the We compared three models based on precision, recall, f1 score, AUC score and accuracy and used two different vectorizers for these three models. As seen from the Table I , when TF-IDF vectorizer was used with the three models, SVC gave best results with accuracy of 0.87 and AUC score of 0.84 . But for all the three models precision was slightly higher than recall which shows that all the three models missed a lot of positive labeled classes but there were also very few false positives meaning whichever positives were detected most of them actually belonged to the positive class.

Table- II: Count Vectorizer Results

\begin{tabular}{llllll}
\hline & Precison Recall & $\begin{array}{l}\text { f1 } \\
\text { Score Score }\end{array}$ & Accuracy \\
& & \multicolumn{5}{c}{ SUC } \\
\hline $\begin{array}{l}\text { Logistic } \\
\begin{array}{l}\text { Regression } \\
\text { Multinomial }\end{array}\end{array}$ & 0.77 & 0.78 & 0.79 & 0.79 & 0.79 \\
NB & 0.83 & 0.88 & 0.88 & 0.90 \\
SVC & 0.83 & 0.83 & 0.83 & 0.83 & 0.85 \\
\hline
\end{tabular}

Table- III: Confusion Matrix Interpretation

\begin{tabular}{lllll}
\hline & TP & FP & TN & FN \\
\hline SVC & 8 & 3 & 23 & 5 \\
Multinomial NB & 11 & 2 & 24 & 2 \\
\hline
\end{tabular}

With TF-IDF vectorizer correctly classifies positive and negative classes and the number of false positives and false negatives are very less. So using these values precision and recall will be calculated and since true positives and true negatives are high and false positives and false negatives are less, it is expected that these two classifiers will have high precision and recall values which can be observed from Table I and Table II . For count vectorizer, Multinomial Naive Bayes gives best results with AUC score of 0.88 and accuracy 0.90. Also, for logistic regression precision is slightly higher than recall and for Multinomial Naive Bayes and Support vector classifiers both precision and recall are high. Hence, except logistic regression, the other two classifiers classify almost all the positive labeled classes correctly. Overall, Multinomial Naive Bayes with count vectorizer gives best results as it gives high precision and recall and has the highest AUC score and accuracy.

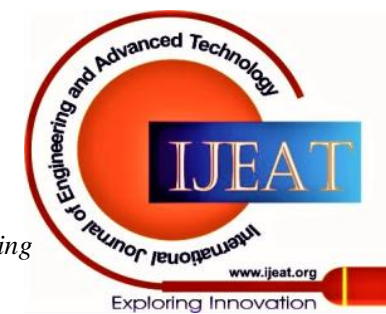



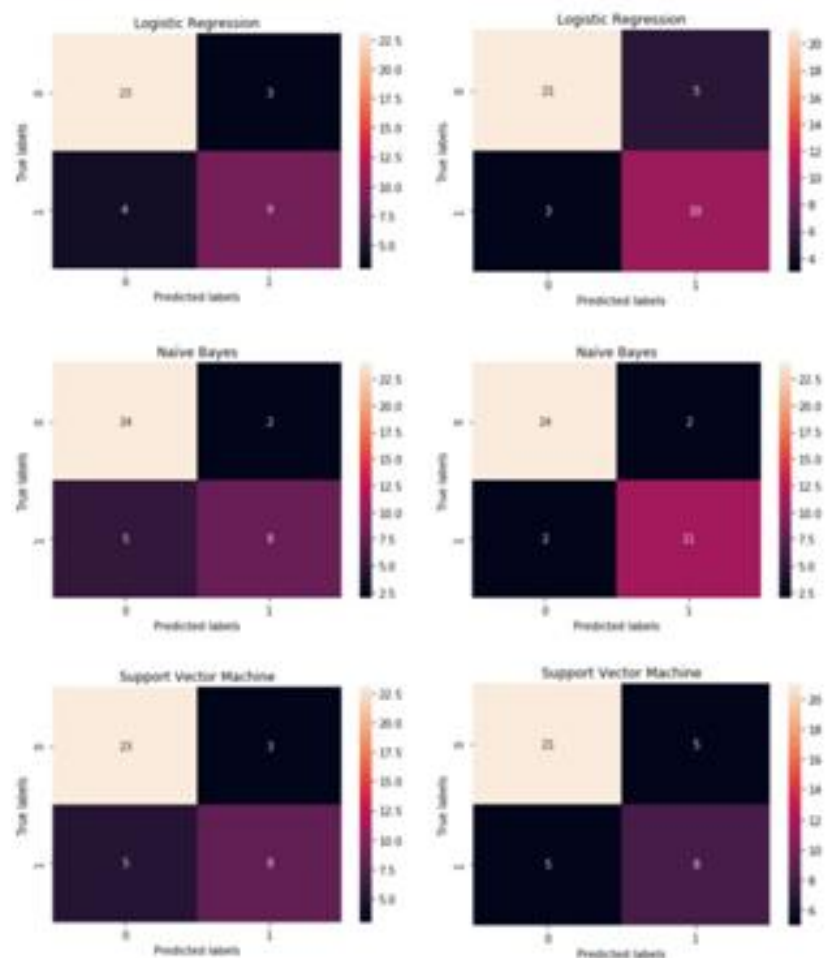

Fig. 7. Confusion Matrix

From the Table III , it is observed that Support Vector Classifier with count vectorizer and Multinomial Naive Bayes

\section{CONCLUSION}

In this paper, we studied and analyzed transcripts thereby classifying it under early signs of depression or serious after- effects of prolonged depression based on the nature of the patient's response. We performed an elaborate study on natural language processing methods for transcript analysis and machine learning algorithms in the field of mental health and obtained satisfactory results with an accuracy of $90 \%$. It was observed that multinomial Naïve Bayes with count vectorizer outperformed logistic regression, support vector machines based on accuracy, F1 score, precision, and AUC score. We also observed that lemmatization yielded better results in comparison to stemming for transcript analysis. The insights that were generated from the dataset were visualized with the help of word clouds and graphs. The word cloud represented some of the most common and frequent words that were common to specific mental healthrelated topics and issues such as trauma, depression, anxiety, etc. This in turn helped us gain a better understanding of the mental health- related issues and the way they are communicated.

\section{FUTURE SCOPE}

Future work could involve diagnosis and classification along with suggestions for treatment that could be recommended based on the transcripts of counseling sessions. The creation of such a platform by leveraging the use of technology and natural processing techniques would help treat mental health issues and create awareness about the same. Such a platform would not only help in identifying mental health issues at an early stage but also reduce the cost. Owing to the current COVID 19 pandemic, there is a lot of uncertainty which makes it all the more important to address mental health. Such research could pave the way for improving counseling and therapy sessions and be an important analysis tool for therapists.

\section{REFERENCES}

1. "Mental disorders affect one in four people." [Online]. Available: https:/www.who.int/whr/2001/mediacentre/ pressrelease/en/

2. M. Khondoker, R. Dobson, C. Skirrow, A. Simmons, and D. Stahl, "A comparison of machine learning methods for classification using simulation with multiple real data examples from mental health studies," Statistical Methods in Medical Research, vol. 25, no. 5, 2016, pp. 1804-1823. [Online]. Available: 10.1177/0962280213502437;https: //dx.doi.org/10.1177/0962280213502437

3. S. \&amp; Braithwaite, C. \&amp; Giraud-Carrier, J. \&amp; West, Barnes, and C. Hanson, "Validating Machine Learning Algorithms for Twitter Data Against Established Measures of Suicidality," JMIR Mental Health, 2016. [Online]. Available: 10.2196/mental.4882

4. M. Deshpande and V. Rao, "Depression detection using emotion artificial intelligence," in 2017 International Conference on Intelligent Sustainable Systems (ICISS), Palladam, 2017, pp. 858-862.

5. M. R. Islam, M. A. Kabir, and A. Ahmed, "Depression detection from social network data using machine learning techniques," Springer Health Information and Science Systems, vol. 6, 2018.

6. Jelodar, Y. Wang, R. Orji, and H. Huang, "Deep Sentiment Classification and Topic Discovery on Novel Coronavirus or COVID19 Online Discussions: NLP Using LSTM Recurrent Neural Network Approach," IEEE Journal of Biomedical and Health Informatics, 2020.

7. S. Butala, A. Lad, H. Chheda, M. Bhat, and A. Nimkar, "Natural Language Parser for Physician's Handwritten Prescription," in 2020 International Conference on Emerging Trends in Information Technology and Engineering, Vellore, India, 2020, pp. 1-7.

8. R. G. Jackson, R. Patel, N. Jayatilleke, A. Kolliakou, M. Ball, G. Gorrell et al., "Natural language processing to extract symptoms of severe mental illness from clinical text: the Clinical Record Interactive Search Comprehensive Data Extraction (CRIS-CODE) project," BMJ Open, vol. 7, no. 1, 2017, pp. e012 012-e012 012. [Online]. Available: $\quad$ 10.1136/bmjopen-2016-012012; https://dx.doi.org/10.1136/bmjopen-2016-012012

9. L. Cook, A. M. Progovac, P. Chen, B. Mullin, S. Hou, and E. BacaGarcia, "Novel Use of Natural Language Processing (NLP) to Predict Suicidal Ideation and Psychiatric Symptoms in a Text-Based Mental Health Intervention in Madrid," Computational and Mathematical Methods in Medicine, vol. 2016, 2016, pp. 1-8. [Online]. Available: 10.1155/2016/8708434;https: //dx.doi.org/10.1155/2016/8708434

10. A. Rumshisky, M. Ghassemi, T. Naumann, P. Szolovits, V. M. Castro, T. H. McCoy et al., "Predicting early psychiatric readmission with natural language processing of narrative discharge summaries," Translational Psychiatry, vol. 6, no. 10, 2016, pp. e921e921. [Online]. Available: 10.1038/tp.2015.182;https: //dx.doi.org/10.1038/tp.2015.182

11. "CounselChat data." [Online]. Available: chat/data/counselchatdata.csvhttps://github.com/ nbertagnolli/counsel-chat/tree/master/data

\section{AUTHORS PROFILE}

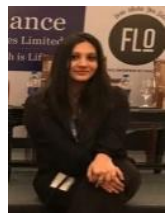

Sarah Hawa is currently a final year student in the Electronics and Telecommunications Department of SPIT, Mumbai. Her research interests are Machine learning, Natural Language Processing and Marketing.

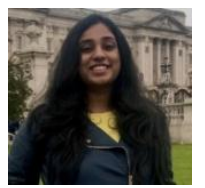

Shriya Akella is currently a final year student in the Electronics and Telecommunications Department of SPIT, Mumbai.

\section{Published By:} Blue Eyes Intelligence Engineering

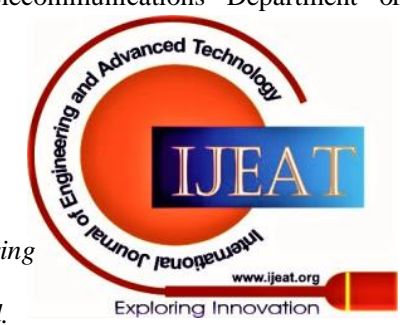


Her research interests lie in Machine learning, Computer Vision, Data Science and Embedded Systems.

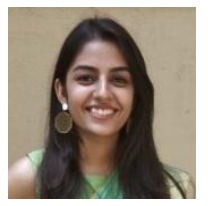

Shrishti Kaushik is currently a final year student in the Electronics and Telecommunications Department of SPIT, Mumbai. Her research interests include Data Science, Machine Learning and Artificial Intelligence.

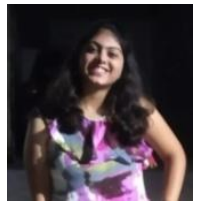

Vrushali Joshi is currently a final year student in the Electronics and Telecommunications Department of SPIT, Mumbai. Her research interests include Data Analytics, Natural Language Processing and Machine Learning.

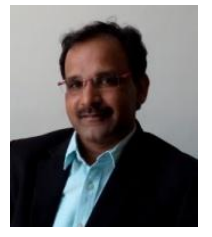

Dhananjay Kalbande is currently a Professor in Computer Engineering and Dean(Industry Relations), Sardar Patel Institute of Technology, Andheri (West),Mumbai, India. He was Head of the Department from April 2012 to Oct 2019. He completed his B.E. in Computer Technology from Nagpur University in 1997 and Master of Engineering in Information Technology in May 2005, from Vivekanand Education Society's Institute of Technology(VESIT), Mumbai University, Mumbai, India. He has obtained a Ph.D in Technology from University of Mumbai, Mumbai in 2011. He has been awarded a Post-Doctorate (PDF)from Tata Institute of Social Sciences(T.I.S.S.) in 2016. He has also been honored as a Senior Research Fellow(SRF) on the NCW-TISS Project,funded by National Commission for Women,Govt.of India at T.I.S.S ,from July 23,2016 to Oct 10, 2016. He was a Research Fellow on the CliX Project at T.I.S.S. ,funded by Tata Trust and M.I.T.(U.S.A.) from Feb 20,2017 to May 19,2017. He has over 19+ Years of experience in teaching \& research. His research interests include Soft computing (Neural Networks, Fuzzy Logic),Computer Network, Human Machine Interaction Decision making and business Intelligence,Mobile application development for social cause. ICT for semi rural development for social causes. He has authored five books namely Graphical User Interface(Pareen Publications), MIS(Pareen Publications), Human Machine Interaction(Wiley Publications), Digital Forensic(Wiley Publications) and Internet of Everythings (StarEdu solutions). He has delivered and conducted workshops, seminars, Tutorials and Expert Talks on NS2, Neural Network, VB.Net and ADO.Net, Transfer Learning.

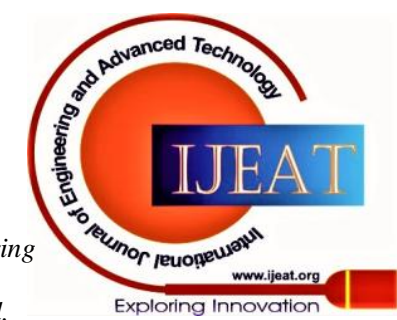

